\title{
Labyrinthe
}

13 | 2002

Numéro 13

Valeurs du territoire

\section{Les territoires de la maison paysanne : vers une dépossession (consentante) du pays?}

Pierre Bourlier

\section{(2) OpenEdition}

Journals

Édition électronique

URL : http://journals.openedition.org/labyrinthe/1536

DOI : $10.4000 /$ labyrinthe.1536

ISSN : 1950-6031

Éditeur

Hermann

Édition imprimée

Date de publication : 15 novembre 2002

Pagination : 51-68

\section{Référence électronique}

Pierre Bourlier, «Les territoires de la maison paysanne : vers une dépossession (consentante) du

pays ? », Labyrinthe [En ligne], 13 | 2002, mis en ligne le 19 mars 2007, consulté le 20 avril 2019. URL http://journals.openedition.org/labyrinthe/1536 ; DOI : 10.4000/labyrinthe.1536

Ce document a été généré automatiquement le 20 avril 2019.

Propriété intellectuelle 


\title{
Les territoires de la maison paysanne: vers une dépossession (consentante) du pays?
}

\author{
Pierre Bourlier
}

1 La présente contribution est un développement d'une recherche dont l'achèvement est prévu d'ici à l'automne 2002: "Maisons des villes, maisons des champs. Figures de la maison individuelle en milieu rural ${ }^{1}$. " Cette étude s'interroge sur l'habitat de la population d'ascendance rurale d'une petite commune de l'Orne, Ceton, et peut constituer une introduction à une histoire des représentations paysannes de la maison. L'histoire des valeurs qui sont associées à cette dernière peut constituer une entrée intéressante ; comme celle des valeurs associées aux territoires de la maison. C'est ce que cet article cherche à explorer.

2 Un précédent numéro de Labyrinthe exposait avec clarté le problème de la définition du (des) territoire(s) et sa relation avec la notion de valeur ${ }^{3}$. En effet, on peut constater que les notions de valeur et de territoire sont intimement liées, mais sont aussi variables. Elles mêlent les échelles de perception subjective et objective; les dimensions politique, économique et symbolique. 


\section{automne-hiver 2002 - Labyrinthe}

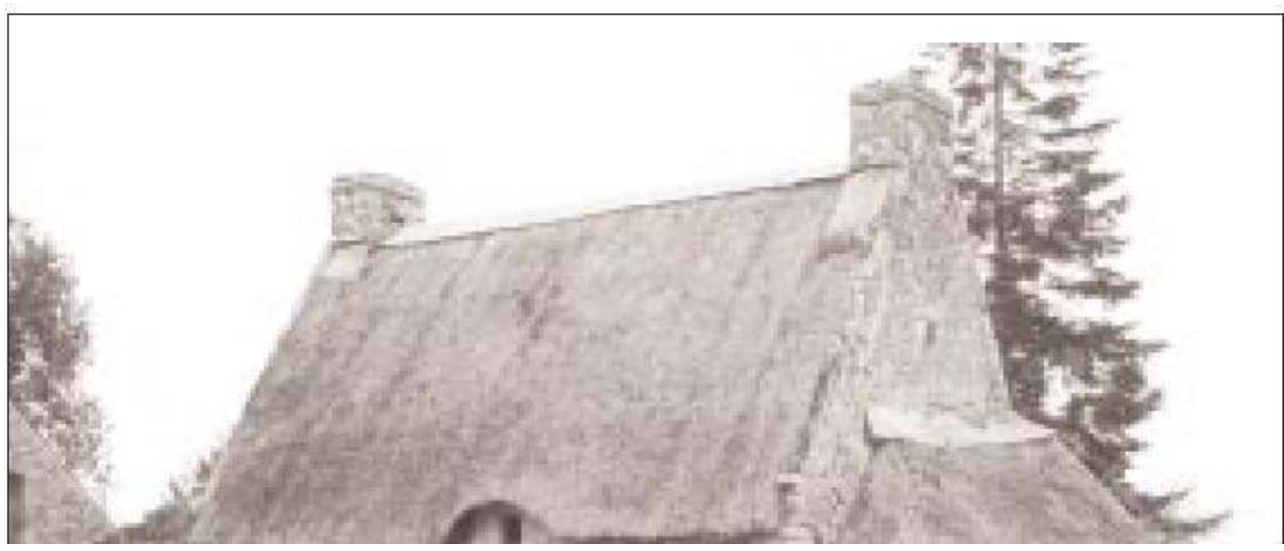

Les maisons paysannes du Perche, Paris, Eyrolles, coll. « Maisons Paysannes de France » (dir. Chantal Pontvianne), 1994

3 Ainsi, pour le sens commun par exemple, le territoire de la « campagne » est associé au terroir $^{4}$; et plus récemment, avec l'émergence des parcs naturels régionaux (PNR), il se distingue mal de la notion un peu floue de paysage. Pour ce qui est de la dimension agricole des campagnes, le territoire est davantage envisagé comme un bien de production parcellisé, souvent perçu comme un élément perturbateur de l'environnement ${ }^{5}$, alors qu'il est pourtant, de fait, structurant.

4 À ces « perceptions » différentes, s'associent des conceptions variables de la valeur. Dans le premier cas, le territoire est qualifié par sa valeur environnementale, pittoresque ou touristique, d'histoire ou d'ancienneté, et relève alors du registre patrimonial. Dans le second cas, le sens commun associe au territoire une valeur de bien de travail et de produit. L'exploitation - c'est-à-dire la ferme et les terres - est à la fois une parcelle de territoire et un «outil de travail » qui peut être évalué en tant que produit ${ }^{6}$ : de par sa localisation, sa "viabilité », ses équipements, le territoire de l'exploitation a bien une valeur marchande.

Mais ce sens commun ne rend pas compte des représentations paysannes du territoire, et ce serait une erreur de penser qu'elles se réduisent à un outil de production. Il possédait et possède une autre dimension. Celle-ci peut être prise en charge par le terme de «Pays ». Nous préfèrerons ici cette notion bien que sa définition contemporaine soit des plus floues. Le rural d'origine l'habite, il est du Pays, ce qui fait de lui - au sens propre - un paysan; alors que l'on ne dit pas être de, ou habiter le territoire. Manière d'affirmer que l'on est d'abord paysan, ce qui est différent du statut socioprofessionnel d'exploitant ${ }^{7}$. Ce terme vient plus spontanément dans les entretiens et conserve une connotation affective et vivante dont le territoire semble totalement dépourvu.

6 L'actualité électorale récente peut introduire la problématique générale de cet article. En effet, à l'image de nombreuses circonscriptions rurales, le canton du Theil-sur-Huisne, qui comprend la commune de Ceton, s'est majoritairement exprimé en faveur du Front national au premier tour de l'élection présidentielle du 21 avril 2002. Le Perche, qui inclut ce canton, comptabilise le plus fort pourcentage de voix pour Jean-Marie Le Pen en Basse- 
Normandie (les deux tours confondus). Comme c'est le cas pour beaucoup d'autres localités, on ne relève pourtant pas dans le Perche de motif tangible qui justifierait une adhésion au programme de l'extrême droite. Il convient de replacer ce vote dans son contexte rural. Des motivations bien éloignées des contenus idéologiques du parti en question ne seraient-elles pas à son origine ? Si ce choix est nationaliste dans un certain sens, est-il réellement « frontiste »?

7 Ne peut-on discerner au travers de ce vote une forme de réaction à ce que l'on pourrait appeler un sentiment de dépossession (consentante) du Pays? Deux observations apporteront un début de réponse. On peut d'abord avancer l'hypothèse qu'il y a eu dans ce choix une confusion d'échelle qui est propre à la notion même de territoire : la "sauvegarde » des valeurs et des ressources nationales a été assimilée à la « sauvegarde » du (des) Pays.

La seconde observation est moins simple. Elle révèle une confusion d'objet : ce n'est peutêtre pas un hasard si le Perche (assimilé au PNR) s'est prononcé majoritairement en faveur de l'extrême droite, car c'est là que le sentiment de dépossession peut être le plus vif, fût-ce inconsciemment. En effet, le Perche, traditionnellement rural et agricole ${ }^{8}$, est constitué d'une multitude de Pays aux identités bien marquées'. Il est cependant aujourd'hui un lieu d'application d'actions «étrangères » émanant du Conseil régional, des $\mathrm{CAUE}^{10}$, etc., qui peuvent être perçues comme des mesures imposées à ces Pays. C'est donc bien d'une question d'identité qu'il s'agit; et le paradoxe est que le PNR du Perche a été créé récemment (il y a quatre ans) pour tenter de préserver une identité régionale fût-elle une construction idéale d'un terroir bel et bien disparu - que l'on a estimée sérieusement menacée... par les paysans, c'est-à-dire par les habitants des Pays !

Il ne s'agit pas ici de chercher à définir le Pays, bien que cette enquête eût été très instructive. Le propos de cet article est d'éclairer un autre problème. Comme nous l'avons $\mathrm{vu}$, la question du territoire et de ses valeurs est problématique en soi, mais aussi parce qu'elle dépend des acteurs concernés. Ces questions sont encore plus délicates à saisir lorsque l'on veut les aborder sous l'angle des représentations. En matière de sources écrites, on conviendra que la population d'ascendance rurale est peu loquace sur ce sujet ${ }^{11}$, mais cela ne veut pas dire qu'elle ne s'exprime pas. Un regard plus attentif permet de constater qu'il existe un certain nombre de témoins de ces représentations. La maison peut être envisagée comme un d'entre eux.

Il s'agit donc d'explorer en quoi les pratiques paysannes de la maison individuelle peuvent être révélatrices des rapports qu'entretiennent les habitants d'ascendance rurale avec le Pays. On voudrait montrer en particulier combien ces pratiques - pour l'histoire récente - ont participé de cette dépossession du Pays, et pourquoi elle fut en partie consentante. Cette étude est exploratoire, elle se donne comme objectif de construire l'hypothèse principale suivante : le processus de rejet de l'habitat traditionnel par les paysans engagé et accepté depuis plus de trente ans est une cause profonde de ce sentiment de dépossession $d u$ Pays, sentiment qui peut être interprété comme la dernière phase d'un déclin de la France rurale, déclin économiquement et socialement consommé depuis les années 1960.

11 Dans un premier temps, nous examinerons cette idée de dépossession consentante du Pays par l'intermédiaire du "témoin» maison individuelle; dans un second temps, nous tenterons d'exposer les causes du rejet de l'habitat traditionnel.

12 L'étude de la mobilité résidentielle au sein des campagnes est une première clé pour saisir les représentations paysannes du Pays. Or, cette mobilité est aujourd'hui d'une nature totalement différente de l'exode rural, elle est nouvelle et pour cette raison intéressante. 
On peut faire le double constat suivant: premièrement un maintien de la population native au sein des Pays, deuxièmement une mobilité résidentielle au sein de la commune. Le rural peut déménager tout en continuant d'habiter dans les limites de son territoire. Aussi, s'il y a dépossession, elle est pour une grande part symbolique. Notons surtout ce qui différencie cette nouvelle mobilité de l'exode rural: dans les cas étudiés, la mobilité résidentielle est le résultat d'un choix et non celui d'une contrainte économique ou professionnelle; il y a donc également une dimension psychologique en jeu. Afin d'ancrer un peu mieux ces constats dans la réalité, arrêtons-nous un instant sur le terrain de notre étude.

13 La campagne française ne se dépeuple plus. Le recensement national de 1999-2000 a montré que plus de la moitié des Français habitent encore dans leurs communes de naissance, le milieu rural contribuant à maintenir cette stabilité résidentielle. Dans le cas de Ceton aujourd'hui, on peut estimer la population résidente native à plus de $80 \%^{12}$.

D'un autre côté, on ne constate pas non plus un abandon des terres, mais une nouvelle répartition. Ceton a vu son nombre d'exploitations passer en moins d'un siècle de 300 à un peu plus de 30 à ce jour. On retrouve la même proportion dans le partage des terres : d'une dizaine d'hectares par exploitation à un peu plus de cent. Sur les 6000 hectares de la commune, la trentaine d'exploitants se partage un peu plus des trois quarts de la superficie, consacrés à la culture et à l'élevage. La surface de terre moyenne s'est donc exactement multipliée en proportion de la diminution du nombre des exploitations. Le partage des terres s'est effectué sans perte.

S'il y a à la fois maintien de la stabilité résidentielle, maintien des terres cultivées et diminution du nombre d'exploitations, c'est donc aussi que l'on a assisté à une diversification des activités (nouveaux arrivants, reconversions d'anciens agriculteurs, population inactive). Ce qu'il faut retenir de ce rapide aperçu au regard de nos remarques de départ, c'est que s'il y a bien aujourd'hui un sentiment de dépossession il n'est lié, de fait, ni à une émigration communale ni à une dépossession de la terre. Il se situe donc ailleurs. Regardons du côté de l'habitat, de la mobilité résidentielle à l'intérieur même de la commune.

Ceton comptabilise aujourd'hui 850 foyers pour une population totale qui avoisine les 2 000 habitants : 550 sont en agglomération, 300 hors du bourg - la plupart en habitat dispersé. Il faut ajouter à ce nombre environ 160 résidences secondaires essentiellement localisées en campagne. Les 550 maisons du bourg se répartissent de la façon suivante : un peu plus d'un tiers en habitat pavillonnaire, un tiers en logement social individuel ou individuel groupé ; le reste comprend des maisons de bourg, quelques fermes de bourg, des maisons ouvrières du début $\mathrm{du} \mathrm{xx}^{\mathrm{e}}$ siècle qui sont pour la plupart inoccupées. On peut ainsi compter à peu près 400 cas d'habitat individuel récent contre une vingtaine de cas seulement dans les années 1960.

Si Ceton est une commune fortement rurale et agricole, marquée par son habitat dispersé qui accentue l'effet "pays de bocages et de haies", elle ne compte cependant actuellement qu'une trentaine d'exploitations en activité sur le terrain, alors que l'on peut estimer à 300 le nombre de foyers d'ascendance rurale en habitat dispersé au début des années 1960. L'essentiel de la population d'ascendance rurale vit donc aujourd'hui en bourg.

18 On peut donc envisager autrement la notion de "désertification » des campagnes, non pas au sens d'un exode rural, mais au sens d'une mobilité à l'intérieur même de la 
commune; une mobilité caractérisée par un déplacement de l'habitat dispersé vers le bourg. On ne développera pas ici cette question, mais ces trajectoires ont des motivations différentes qui peuvent pourtant être regroupées en quelques catégories, dont certaines «figures» architecturales témoignent. Ainsi, par exemple, le choix d'un pavillon à proximité du bourg peut-il être motivé par un changement d'activité des jeunes ou des moins jeunes, ou par un départ à la retraite. Le pavillon permet alors un accès au mode de vie citadin: ce que l'on pourrait appeler la «maison-appartement» est un vecteur d'acculturation citadine. Plus surprenant, ce choix peut encore prendre la forme d'un « retour à la terre » après une émigration pour motif professionnel, mais en lotissement.

19 L'étude des trajectoires résidentielles permet de mieux comprendre ce sentiment de dépossession consentante du Pays : la tendance depuis un peu plus de trente ans est au rejet de l'habitat traditionnel de la part du paysan. Certes, quelques agriculteurs travaillent encore la terre et habitent sur leur lieu d'activité. De fait, on peut distinguer deux groupes d'exploitations encore en habitat dispersé : le premier groupe relève de l'« esprit d'entreprise ", il concerne les grosses ou moyennes exploitations qui ont su s'adapter grâce aux $\mathrm{Gaec}^{13}$ par exemple. On peut qualifier les membres du second groupe de " résistants ». Il est constitué de petites exploitations familiales anciennes, souvent à la limite des normes sanitaires, qui disparaîtront très probablement à la mort de leurs propriétaires. Cela nous rappelle que nous sommes aujourd'hui à un tournant générationnel dans le mode d'occupation des campagnes par la population d'ascendance rurale.

Relevons donc que, en dehors de ces deux groupes minoritaires, les paysans n'habitent plus le Pays (sinon le bourg qui n'est en quelque sorte que son centre, un point sur le territoire). Et c'est là, me semble-t-il, un constat important pour la sociologie rurale. Si la "dépossession » est "consentante », c'est que cette migration dans la commune relève d'un choix, bien que par certains aspects ce choix ait été contraint et ce, généralement, à l'insu des intéressés. Il s'agit de comprendre les causes de ce choix.

21 C'est volontairement que nous laisserons de côté certaines causes évidentes de la préférence pour le pavillon en bourg : accès aux équipements et aux services, proximité des petits commerces, réduction des trajets automobiles, etc., autant de motivations bien réelles qui relèvent d'une demande de commodité et révèlent la prégnance du modèle citadin, ce qui sera développé un peu plus loin.

La première cause du désamour du paysan pour sa maison est objective. En effet, quel est l'état du patrimoine immobilier dont ont hérité les acteurs du changement résidentiel des années 1960-1970?

23 Pour répondre à cette question, on dispose d'informations très utiles réunies dans les "enquêtes", en particulier pour la première moitié du $\mathrm{xx}^{\mathrm{e}}$ siècle ${ }^{14}$. Ces dernières montrent bien l'état de vétusté du patrimoine immobilier domestique traditionnel. Ainsi, à la veille de la Seconde Guerre mondiale, neuf maisons sur dix ont été construites avant 1915, huit sur dix avant 1871 et six sur dix ont plus d'un siècle.

Les campagnes ont peu bénéficié de l'élan de la reconstruction. C'est surtout dans les années 1960 qu'un renouveau se fait sentir. Il y a, en moyenne, deux fois plus de constructions neuves par an entre 1962 et 1968 qu'entre 1949 et 1953. Mais, plus que les chefs d'exploitations, ce sont les salariés agricoles qui en bénéficient. En 1968, 8 \% seulement des ménages d'agriculteurs habitent une maison dont la construction est postérieure à la guerre; dans les communes rurales même, exploitants comme salariés 
demeurent les catégories sociales qui occupent le plus vieil habitat et le moins bien équipé.

Ainsi pour l'équipement: en 1962, $42 \%$ des logements d'agriculteurs sont à la fois construits en dur, dotés d'une cuisine et équipés d'eau courante à l'intérieur; $9 \%$ ont en outre des cabinets d'aisance intérieurs et $6 \%$ seulement ont, en plus, une baignoire ou une douche installée. Dans la majorité des fermes, on continue d'aller chercher l'eau au puits. Relevons surtout que lorsque des efforts sont consentis, il vont dans le sens de l'amélioration de l'exploitation en tant qu'« outil de travail», et non dans celui de l'amélioration de l'habitat.

Les politiques agricole et d'aménagement de l'espace rural ces quarante dernières années ont-elles contribué à améliorer l'habitat traditionnel paysan? On peut répondre par l'affirmative, mais indirectement puisque l'entretien de ce patrimoine a été essentiellement le fait des résidences secondaires, c'est-à-dire qu'il concerne un habitat « libéré » par sa population résidente initiale. En fait, ces politiques ont surtout contribué à « assainir » le parc des exploitations !

L'évolution démographique révèle un phénomène intéressant, premier signe tangible de cet «assainissement ». Ceton comptait 3300 habitants en 1860, environ 3000 à la fin du siècle dernier, presque 2000 en 1960, un peu plus de 1500 en 1970, date qui marque le plus fort creux démographique du siècle, comme c'est le cas pour d'autres communes rurales de la même taille ${ }^{15}$. En résumé : entre 1860 et 1960, la commune perd $39 \%$ de sa population, et $25 \%$ en seulement quinze ans (entre 1960 et 1975) !La population est remontée rapidement après la création d'une zone industrielle en $1975^{16}$ : on dénombrait 1800 habitants en 1990. Avec l'installation d'une population jeune, la courbe de natalité a également repris sa croissance et la population atteint aujourd'hui presque 2000 habitants. Le creux de la période 1960-1975 est significatif et peut s'expliquer par la coïncidence de plusieurs facteurs. La politique d'orientation agricole est un de ceux-là ${ }^{17}$.

Des mesures nouvelles, volontaristes et progressistes tant du point de vue agricole que de celui de l'aménagement de l'espace rural ont été mises en œuvre dès les débuts de la Ve République. Pour vendre des produits alimentaires permettant d'acheter les matières premières qui lui font défaut, la France a dû intensifier sa production agricole et devenir exportatrice. Il a fallu encourager la mécanisation des exploitations afin que la campagne participe à la reprise industrielle. Mais ce projet d'envergure supposait bien sûr d'être en mesure de libérer de la surface, de restructurer les terres cultivables et donc d'éliminer les moins compétitifs. Les objectifs à court terme étaient définis de la manière suivante : réduire la population d'agriculteurs dans les plus brefs délais, moderniser les exploitations, accélérer et organiser l'exode, fixer un type d'exploitation à responsabilité personnelle, adapter l'agriculture au capitalisme national, rationaliser l'appareil productif.

Cinq outils des lois d'orientation de 1960 nous intéressent ici au premier chef :

$1^{\circ}$ La définition d'une « unité de production viable » au cas par cas, par département. Ses caractéristiques sont les suivantes: afin de faire de chaque paysan un petit chef d'entreprise, il fallait lui faciliter l'accès à la propriété. L'unité d'exploitation " moyenne " est celle qui utilise le plus de produits industriels, et qui peut donc être pleinement considérée comme un soutien à l'industrie française. Enfin, la dernière caractéristique de l'« unité de production viable » est le reflet d'une réalité sociologique remarquable dans les années 1960: elle doit pouvoir fonctionner avec deux "unités de travailleurs- 
hommes ", ce qui correspond à la fin du modèle de la famille patriarcale et à l'émergence de celui du couple d'agriculteurs ${ }^{18}$.

$2^{\circ}$ La mise en place des Safer ${ }^{19}$ pour superviser, gérer et distribuer le foncier en fonction de la « viabilité » des unités.

$3^{\circ}$ Les prêts sélectifs du Crédit agricole consentis aux « unités viables ».

$4^{\circ}$ La mise en place des Gaec qui permirent le groupement d'exploitations familiales et le cumul des terres.

$5^{\circ}$ Le cinquième outil est celui qui a eu le plus de conséquences au regard de notre propos. Il peut être interprété comme une réponse à cette question: comment éliminer les structures non « viables » sans perdre un électorat utile? Le Fonds d'action sociale pour l'aménagement des structures agricoles a permis d'accélérer le départ à la retraite des agriculteurs grâce au versement d'une indemnité. Cette mesure a d'abord touché les régions défavorisées puis tous les départements.

Ainsi, les « lois d'orientation » ont réussi à accentuer le processus d'élimination des plus faibles. La mesure la plus efficace fut sans conteste l'indemnité viagère de départ. Elle a permis, en quelques années, de libérer 8500000 hectares, soit le quart de la surface agricole utile, attribuée à plus de 500000 agriculteurs.

Cette dernière mesure est d'une importance fondamentale pour comprendre le rejet de la maison traditionnelle et les migrations à l'intérieur du Pays. Un paysan qui quitte l'exploitation parce qu'elle n'est pas viable va voir - contre une indemnisation - ses terres redistribuées à ses pairs par l'intermédiaire de la Safer. Mais que faire des bâtiments d'exploitation et d'habitation? En dehors du problème psychologique et culturel posé par le fait d'habiter une ferme sans terre, il faut bien comprendre que le paysan a tout intérêt à satisfaire un marché où la demande en habitat traditionnel va croissant pendant ces mêmes années : les résidences secondaires.

On l'a vu, les années 1960-1970 sont caractérisées par la coïncidence de trois facteurs : des mesures politiques nouvelles, le développement du pavillonnaire en proximité de bourg, et le phénomène des résidences secondaires.

Que l'on ne s'y trompe pas, le développement de ce nouveau mode de résidence est un phénomène récent ${ }^{20}$ et s'accompagne de la construction - au sens figuré comme au sens propre - d'une campagne idéale vue comme une alternative aux excès de l'urbanisation, et qui contribue à entretenir l'idée encore vivante de la paysannerie gardienne des valeurs traditionnelles et du passé. Mais alors que ce discours idéologique se généralisait dans la société à la fin des années 1960 et dans les années 1970, le discours dominant de la politique agricole en différait radicalement. L'heure était aux jeunes, à la modernité et au mérite.

De nombreuses recherches ont exploré les diverses facettes du phénomène des résidence secondaires ${ }^{21}$. Notons ici que loin de s'en tenir à une simple occupation ponctuelle sur les " territoires vert », ces nouveaux modes de résidence ont eu - et continuent d'avoir - des conséquences importantes sur la configuration des campagnes. Ils deviennent une nouvelle force qui a le pouvoir d'imposer une représentation (idéale) du Pays sur le pays. Ainsi que l'avaient relevé Michel Gervais, Marcel Jollivet et Yves Tavernier dans l'Histoire de la France rurale, les résidents de week-end deviennent les défenseurs actifs du terroir :

«La défense du " pays » s'identifie forcément à celle de la mise en valeur de ces nouvelles ressources ou richesses «naturelles » que sont la végétation, le paysage, l'habitat et l'espace lui-même, dans ces zones dont l'industrialisation est exclue. Et 
précisément parce qu'il s'agit de la terre (des terroirs), de l'habitat traditionnel, de tout ce qui porte la trace de la civilisation du "pays", la revendication du développement peut prendre un aspect culturel et régionaliste. [...] Dans cette perspective, le monde rural n'est plus, pour le régime politique, le dernier carré de la garde, mais simplement un grand musée vivant exemplaire, lieu de cure reconstituante à l'usage des citadins ; cette finalité s'exprime tout particulièrement dans la création des parcs régionaux et nationaux ${ }^{22}$.» exemple illustre assez bien les moyens de construction de cette campagne «idéale » : les documents conseils. Le développement de ces documents est contemporain de la coïncidence des trois facteurs identifiés précédemment. Ils apparaissent parallèlement à la création des $\mathrm{CAUE}^{23}$, d'associations que l'on pourrait qualifier d'« engagées », telles Maisons paysannes de France ${ }^{24}$, ou la Société des amis du Perche (qui a d'ailleurs son siège à Ceton), ainsi que des musées départementaux des Arts et Traditions populaires. Ces organismes prennent part dans la diffusion de ces documents et sont bien souvent à l'origine de leur rédaction.

41 Ce " genre » littéraire renseigne particulièrement bien sur la stratégie de diffusion d'un modèle idéal de la maison rurale traditionnelle qui conforte et renforce les représentations citadines d'un habitat qui n'a, en fait, jamais existé spontanément. mener ici une étude détaillée de ces documents, relevons simplement quelques caractéristiques communes qui permettent de parler d'un «genre ${ }^{25}$ ». Remarquons d'abord qu'ils sont généralement précédés par une partie introductive qui présente les «éléments » de l'architecture rurale ordinaire (un peu dans la tradition des manuels du type "éléments de l'architecture classique»). Le registre est didactique et le texte agrémenté d'illustrations soignées ; le tout rappelant bien souvent l'ouvrage classique de Georges Doyon et Robert Hubrecht sur l'architecture rurale ${ }^{26}$. Le volume de cette partie introductive dépend de la nature de l'ouvrage. Elle est réduite pour François Toumit qui privilégie la dimension de conseil ; elle constitue l'essentiel du livre de Roger Fischer, qui répond à une ambition historique plus marquée.

points caractérisent les fiches conseils à proprement parler. C'est le système d'opposition binaire qui frappe en premier le lecteur. Les pages sont en vis-à-vis et la symbolique de latéralisation est efficace : à gauche, « ce qu'il faut éviter ", et à droite, « ce qu'il convient de faire ». Ensuite le discours opère toujours sur le mode prescriptif. Enfin le graphisme de la page de droite est notablement plus soigné que celui de la page de gauche.

44 Ces documents semblent bien recourir à une stratégie de communication d'un modèle, qu'elle soit consciente ou non (et l'on peut raisonnablement faire l'hypothèse qu'elle l'est). Elle recourt à un procédé rhétorique qui consiste à produire un discours à teneur doctrinale en partant de genres différents qui captent l'adhésion par leur neutralité.

Pour conclure, revenons sur l'exploitation et précisons l'hypothèse principale de cet article quant au rapport qu'entretient le paysan avec la maison, en approfondissant les trois sens que peut prendre la notion de « distinction ».

Le monde rural est passé en moins d'un siècle d'un domaine coopératif à un ensemble constitué de plusieurs domaines privés, d'une société communautaire à l'individualisation de ses membres, dont le fort désir de maison individuelle est peut-être un corollaire. On constate cependant, effet qui se fait plus sensible depuis trente ans, que le succès de la propriété coïncide avec un rejet manifeste de la maison traditionnelle, rejet qui

Labyrinthe, 13 | 2007 
s'accompagne d'un attrait pour le pavillonnaire. Développons donc l'hypothèse suivante : c'est, paradoxalement, le triomphe de l'exploitation familiale qui est à l'origine du désamour du paysan pour sa maison. Comme nous l'avons vu, cette hypothèse est fondée sur le plan objectif (indemnités de départ, développement du marché des résidences secondaires, etc.), mais elle porte aussi une dimension subjective qu'il ne faut pas négliger et qui peut expliquer l'aspect « consentant » de ce sentiment de dépossession du Pays.

La formule «à chaque famille son exploitation ${ }^{27}$ " revendiquait la propriété et l'autonomie productive. Bénéfique sur le plan professionnel et social pour quelques-uns, elle a fait de l'exploitation un bien de production, de la ferme un outil de travail essentiellement. Avec la mécanisation des tâches, la réduction des actifs à l'exploitation, l'emploi de la femme à plein temps, le départ des enfants, la maison traditionnelle n'est plus ce lieu de réunion où les jeunes comme les vieux avaient leurs rôles, lieu de veillées, de festivités saisonnières et lieu d'inscription des vieilles croyances ${ }^{28}$.

Signe caractéristique de cette transformation : ce sont les salles des fêtes ou foyers ruraux construits dans les années 1950 qui devaient opérer ce déplacement des loisirs de la ferme vers un «foyer", une «salle commune » qui n'est plus la " maison» entendue comme partie de l'exploitation réservée à la vie de la famille. La maison recoupe difficilement désormais ce qui est un droit d'accès à une culture de loisirs largement diffusée par les médias, et dont le modèle des citadins en maisons de week-end ou des « néo-ruraux » est exemplaire.

automne-hiver 2002 - Labyrinthe
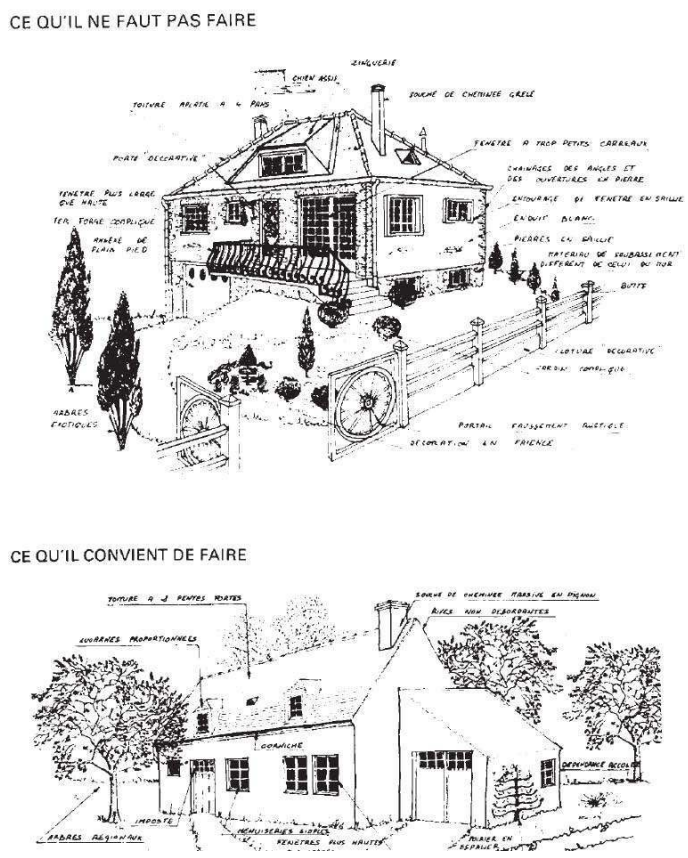

Ces images sont tirées de Comment construire dans le Perche ?, Mortagne-au-Perche, Institut Percheron pour l'habitat rural et urbain avec la participation de la « Conservation Régionale des Bâtiments de France de Basse-Normandie ", l'Association des Amis du Perche (éd.), $\mathrm{n}^{\circ}$ hors série des Cahiers Percherons, préface de Michel Fleury et Philippe Siguret, s.d. (1977).

L'accession au pavillonnaire par une population d'ascendance rurale peut donc d'abord apparaître comme une nécessité ressentie de distinguer l'habitation de l'outil de travail. 
C'est là le premier sens de la « distinction » que permet le pavillon, et qui est à notre avis le plus important. Accéder au pavillonnaire, à la maison « libre », propre et confortable, peut aussi correspondre à un signe de distinction sociale. Il arrive alors que l'on dise que l'on habite « en ville » (qui dit cela ?) [pour dire le bourg], tout en allant les week-ends ou durant les beaux jours "à la campagne », dans ce qui est en fait une «cabane » dans un jardin potager, ou un «chalet». Enfin, on peut interpréter cet attrait pour le pavillon comme un vecteur d'acculturation citadine : il permet d'intégrer un mode vie qui se rapprocherait de celui du citadin, de prendre ses distances de la campagne, du monde paysan, en quelque sorte de se " dépayser ». C'est aussi en cela que si « dépossession » il y a, elle est en partie « consentante».

\section{BIBLIOGRAPHIE}

BROMBERGER Christian, « Pratiques et représentations des espaces bâtis. Habitat et espace dans le monde rural », Ethnologie de la France, 3, Paris, Éditions de la Maison des sciences de l'homme, 1988, pp. 75-86.

FISCHER Roger, Les Maisons paysannes du Perche, Paris, Eyrolles, 1994.

GERVAIS Michel, JOLLIVET Marcel et TAVERNIER Yves, Histoire de la France rurale, 4, Paris, Seuil, 1977.

JOLLIVET Marcel, «Typologie des évolutions démographiques cantonales depuis le début du XIX ${ }^{\mathrm{e}}$ siècle ", Études rurales, 27, juillet-septembre 1967, pp. 72-91.

RAULIN Henri, «L'architecture rurale française : une enquête nationale inédite (1941-1948) », Études rurales, 13-14, 1964, pp. 96 et suivantes.

WEBER Eugen, La Fin des terroirs. La Modernisation de la France rurale, 1870-1914, Paris, Fayard, 1983.

\section{NOTES}

1. Programme « Maison individuelle, architecture, urbanité » initié par le Plan urbanisme construction architecture (Puca) en 1999. Le Puca est une structure de recherche et d'expérimentation rattachée au ministère de l'Équipement, des Transports et du Logement.

2. Afin de distinguer le Pays du pays (au sens du territoire national), le mot commencera toujours avec une majuscule.

3. «Valeurs du territoire », dans Labyrinthe $n^{\circ} 11$, hiver 2002, pp. 27 et suivantes.

4. Mais qu'en est-il du terroir aujourd'hui ? Voir à ce sujet Eugen Weber, La Fin des terroirs. La modernisation de la France rurale, 1870-1914, Paris, Fayard, coll. « Recherches », 1983, (Stanford University Press, 1976).

5. « L'entretien du bocage et de l'habitat rural est contradictoire avec l'industrie agricole qui transforme la campagne et dont l'enjeu majeur est la « rentabilité ». Cette remarque de Jacques Simon dans « La campagne dont le paysan est l'auteur est à réinventer », Pages Paysages, 5, 1994/95, p. 112, est symptomatique de l'idée que l'identité du Pays est 
menacée par les paysans. De même, on peut lire de Joseph Lagisquet, « Du respect de la ruralité des paysages », Maisons paysannes de France, 112, $2^{\mathrm{e}}$ trim. 1994, pp. 25-26. Sur le développement industriel en milieu rural : Industrie et paysage rural. Dommages causés au paysage rural par l'industrie et les remèdes à y apporter, Paris, ministère de l'Agriculture, DARS, s. d. (suit une étude lancée en juin 1972) et Anne Kriegel, Luc Etivant, Bâtiments agricoles et paysages. Éléments pour une réflexion, Trappes, Cnerp, 1976.

6. C'est Albert Demangeon qui a le premier analysé l'exploitation comme maison et comme « outil de travail ». Voir Albert Demangeon, « L'habitation rurale en France. Essai de classification des principaux types ", Annales de géographie, XXIX, septembre 1920. - La Définition et le classement des maisons rurales, Paris, Denoël, exposition de 1937.

7. On notera que cette réévaluation du terme de paysan est relativement récente. À ce sujet, voir P. Barral, « Note historique sur l'emploi du terme paysan », Études rurales, 21, 1966, pp. 72 et suivantes.

8. Prenons pour exemple la commune de Ceton qui compte 2000 habitants pour une surface de 6000 hectares.

9. Parmi les quelques ouvrages et articles traitant de l'identité architecturale du Perche, citons le livre de Roger Fischer sur lequel nous aurons l'occasion de revenir, Les Maisons paysannes du Perche, Paris, Eyrolles, coll. « Maisons paysannes de France » (dir. Chantal Pontvianne), 1994.

10. Conseils d'architecture, d'urbanisme et de l'environnement.

11. La recherche s'est pourtant intéressée aux représentations paysannes. Citons par exemple Christian Bromberger, « Pratiques et représentations des espaces bâtis. Habitat et espace dans le monde rural ", dans Ethnologie de la France, cahier 3, Paris, Éditions de la Maison des sciences de l'homme, 1988, pp. 75-86 et l'article de Monique Vincienne, « Un aspect de l'exode rural : l'image de la ville chez les immigrés », Études rurales, 18, 1965, pp. 79-100, qui répertorie trois types d'image de la ville : « spontanée », « réfléchie », « comparée ».

12. En l'état d'avancement de la recherche, les sources privilégiées sont qualitatives et de trois sortes : les données disponibles en mairie, les entretiens individuels non directifs, les entretiens auprès d'informateurs (j'en profite ici pour remercier chaleureusement $\mathrm{M}$.

Raymond Guillemin, adjoint au maire, ainsi que M. Louis Géhan pour leur disponibilité et leurs conseils avisés).

13. Groupements agricoles d'exploitation en commun.

14. En partie grâce à l'impulsion donnée par les hygiénistes, les enquêtes sur l'habitation ont connuun certain succès dans la seconde moitié du XIX ${ }^{e}$ siècle. Citons par exemple $\mathrm{A}$.

de Foville, Enquête sur les conditions de l'habitation en France. Les maisons types, Paris, Leroux, 1894-1899, 2 vol. Concernant le milieu du xxe siècle, nous nous référons ici à l'Enquête sur l'habitation rurale en France, Paris, H. Dannaud, 1939 ainsi qu'à l'« Enquête folklorique sur l'habitation rurale en France effectuée par des équipes de jeunes architectes (chantier intellectuel) pour le Musée national des Arts et Traditions populaires et sous la haute direction du conservateur M. Georges-Henri Rivière », 1943-1944. Voir un compte rendu dans Techniques et Architecture, 11-12, novembre-décembre 1943 et l'article de Henri Raulin, «L'architecture rurale française : une enquête nationale inédite (1941-1948) », Études rurales, 13-14, 1964, p. 96 et suivantes, pour un historique de ce chantier.

15. Voir Marcel Jollivet, « Typologie des évolutions démographiques cantonales depuis le début du XIX siècle », Études rurales, 27, juillet-septembre 1967, pp. 72-91.

16. Deux industries comptabilisant un peu moins de 200 emplois, auxquels il faut ajouter une cinquantaine d'emplois dans le secteur artisanal : machinerie, serrurerie, peinture. 
17. Sur ces questions, voir « La politique agricole à l'heure du capitalisme mondial », Histoire de la France rurale, 4, Paris, Seuil, 1977, pp. 625 et suivantes.

18. Voir M. Segalen, Mari et femme dans la société paysanne, Paris, Flammarion, 1980.

Associations des ruralistes français, La Pluriactivité dans les familles agricoles, ARF édition, 1984. Souvenons-nous aussi du film d'Yves Robert, Alexandre le Bienheureux (1967), qui illustrait avec autant de bonhomie que d'ironie cette nouvelle identité de l'exploitation 19. Sociétés d'aménagement foncier et d'établissement rural.

20. En 1966, une première évaluation aboutit au nombre de 4400000 personnes. En 1970, la même évaluation donne 7066000 personnes. Selon l'enquête communale de 1969-1970, la population saisonnière est encore plus importante, puisqu'elle atteint 7825000 personnes (Histoire de la France rurale, op. cit., p. 404).

21. Voir L. Torrion, «L'aménagement rural en France ", Le Moniteur des travaux publics et du bâtiment, 6, 8 février 1975. Les Résidences secondaires en France dans le cadre de l'habitat de loisir, La Documentation française, 9 novembre 1972. Christine Dourlens, Pierre VidalNaquet, « Les transformations des filières de production de l'habitat rural : le cas des résidences secondaires ", Rapport DGRST.

22. Histoire de la France rurale, op. cit., pp. 413-414.

23. Les CAUE présents sur la quasi-totalité des départements sont issus de la loi sur l'architecture du 3 janvier 1977.

24. L'association Maisons paysannes de France - comme sa revue - fut fondée en 1965 et reconnue d'utilité publique en 1985. Elle compte 8000 adhérents et 70 délégations départementales, dont l'Orne et le Pays d'Ouche-Perche, toutes deux représentées par sa présidente, Chantal Pontvianne. Outre sa revue, l'association couvre différentes actions dont le concours René Fontaine pour la restauration de maisons paysannes.

25. Pour ce qui suit, nous ne nous référons qu'aux titres suivants, qui donnent une bonne idée de ces documents des années 1970 à aujourd'hui : François Toumit, Comment construire dans le Perche?, Mortagne-au-Perche, Institut percheron pour l'habitat rural et urbain avec la participation de la Conservation régionale des bâtiments de France de Basse-Normandie, l'Association des amis du Perche (éd.), hors série des Cahiers percherons, préface de Michel Fleury et Philippe Siguret, s. d. (1977) ; Roger Fischer, op. cit.; Le Perche, fiches architecturales et paysagères. Valoriser le patrimoine, CAUE d'Eure-et-Loire, CAUE de l'Orne, parc naturel régional du Perche, s. d. (1999-2000).

26. L'Architecture rurale et bourgeoise en France, Paris, Vincent Fréal et Cie, 1941.

27. Expression de Victor Boret à la Chambre des députés le 3 juin 1919.

28. Voir « L'entrée dans la modernité. Fêtes et coutumes en déclin », dans Histoire de la France rurale, op. cit., pp. 354-362.

\section{RÉSUMÉS}

Cet article tente d'explorer en quoi les pratiques paysannes de la maison individuelle peuvent témoigner des rapports qu'entretiennent les habitants d'ascendance rurale avec leur territoire aujourd'hui. Plus particulièrement, il voudrait examiner l'hypothèse suivante : le processus de rejet de l'habitat traditionnel par les paysans engagé et accepté depuis plus de trente ans est une 
cause profonde de ce que l'on pourrait appeler un « sentiment de dépossession (consentante) du Pays », sentiment qui peut être interprété comme la dernière phase d'un déclin de la France rurale, déclin économiquement et socialement consommé depuis les années 1960.

Territorial Values

The peasant home and its land : towards a (consenting) dispossession of the Country?

This article explores how single family housing practices among the peasantry can serve as a window from which to examine the relations that modern rural inhabitants have with their property. More specifically, it will examine the following hypothesis: the process of the rejection of the traditional habitat engaged in and accepted by peasants in the last three decades is an important cause of what one might call a "sentiment of dispossession in agreement with the Country", a sentiment that could be interpreted as the last phase of the economic and social decline of rural France that has occurred since the 1960's.

\section{AUTEUR}

\section{PIERRE BOURLIER}

Pierre Bourlier est architecte. Il poursuit depuis 1998 une thèse de doctorat en urbanisme et aménagement, mention architecture (université Paris VIII) : « Le Style et l'ornement. Aux sources d'une théorie moderne de l'architecture. XIX ${ }^{\mathrm{e}}$ siècle. » Parallèlement à cette recherche, Pierre Bourlier est enseignant vacataire à l'École d'architecture PierreMalaquais. Il est également le co-auteur d'un site Internet de valorisation et de diffusion de la recherche architecturale et urbaine en France pour le compte du Plan urbanisme construction et architecture du ministère de l'Équipement ; actuellement, il est chargé par l'Institut français d'architecture-Chaillot, Cité de l'architecture et du patrimoine de la coordination européenne du programme Gaudi (Gouvernance, architecture, urbanisme : une démocratie interactive). 